Pacific Journal of Mathematics

A NOTE ON COUNTABLY SUBPARACOMPACT SPACES 


\title{
A NOTE ON COUNTABLY SUBPARACOMPACT SPACES
}

\author{
THOMAS R. KRAMER
}

It is the purpose of this paper to characterize countably subparacompact spaces in a number of ways and to point out similarities in the pathologies of countably subparacompact spaces and normal spaces. It will be shown inter alia, that a space is countably subparacompact if and only if it is countably $\sigma$-paracompact, and also if and only if it is countably metacompact and subnormal. The well known product of ordinal spaces, $W \times W^{*}$, is shown to be not countably subparacompact, despite the fact that $W^{*}$ is compact and $W$ is countably subparacompact and normal.

1. Introduction. Countably subparacompact spaces were first defined in the literature by R. E. Hodel in [3] as follows: a topological space is countably subparacompact iff every countable open cover of it has a $\sigma$-discrete closed refinement. The concept had been briefly studied in an earlier paper [7] by M. Mansfield. He showed that in normal spaces, countable subparacompactness is equivalent to countable metacompactness. Recall that a space is countably metacompact iff every countable open cover of it has a point finite open refinement. The following result of Hodel in the work cited above extended Mansfield's theorem: every countably subparacompact space is countably metacompact. A number of further results were developed independently by the author [6] and M. K. Singal and P. Jain [8].

We shall use the following conventions. The end of a proof is denoted by $\square$, the positive integers by $N$, and implication by $\Rightarrow$. "Iff" means "if and only if". $X$ and $Y$ are always topological spaces.

2. Characterizations of countably subparacompact spaces.

THEOREM 2.1. The following are equivalent.

(a) Every countable open cover of $X$ has a $\sigma$-discrete closed refinement (i.e., $X$ is countably subparacompact).

(b) Every countable open cover of $X$ has a $\sigma$-locally finite closed refinement.

(c) Every countable open cover of $X$ has a $\sigma$-closure preserving closed refinement.

(d) Every countable open cover of $X$ has a countable closed refinement.

In [8] Singal and Jain give this theorem with only parts (a)-(c), and the proof offered is consequently somewhat intricate. The inclusion 
of part (d) simplifies matters.

Proof of Theorem 2.1. (a) $\Rightarrow(\mathrm{b}), \quad(\mathrm{b}) \Rightarrow$ (c), and (d) $\Rightarrow$ (a) are obvious. To see $(\mathrm{c}) \Rightarrow(\mathrm{d})$, suppose $\left\{U_{n}: n \in N\right\}$ is a countable open cover of $X$ with $\left\{F_{m \alpha}: \alpha \in A_{m}, m \in N\right\}$ as a $\sigma$-closure preserving closed refinement $\left(\left\{F_{m \alpha}: \alpha \in A_{m}\right\}\right.$ is a closure-preserving collection of closed sets for each $m \in N)$. Then letting $G_{m n}=\bigcup\left\{F_{m \alpha}: \alpha \in A_{m}, F_{m \alpha} \subset U_{n}\right\}$, it is clear that $\mathscr{G}=\left\{G_{m n}: m, n \in N\right\}$ is the required countable closed refinement.

In D. K. Burke's paper [1, p. 655] it was shown that $\sigma$-paracompactness (a definition introduced by Arhangelskii) is equivalent to subparacompactness. It is the case that if we define countable $\sigma$ paracompactness in the obvious way, the analogous theorem is true.

DEFINITION 2.2. $X$ is countably $\sigma$-paracompact iff given a countable open cover $\mathscr{C}$ of $X$, there is a sequence $\left\{\mathscr{C}_{n}\right\}$ of open covers of $X$ such that given $x \in X$, there are $n \in N$ and $U \in \mathscr{U}_{\text {with }} s t\left(x, \mathscr{U}_{n}\right) \subset U$.

THEOREM 2.3. $X$ is countably subparacompact iff $X$ is countably $\sigma$-paracompact.

Proof of Theorem 2.3. " $\Longrightarrow$ " Let $\mathscr{C}$ be a countable open cover of $X$ with countable closed refinement $\left\{F_{n}: n \in N\right\}$. For each $n \in N$ let $U_{n}$ be an element of $\mathscr{U}_{\mathcal{C}}$ with $F_{n} \subset U_{n}$ and let $\mathscr{U}_{n}=\left\{U_{n}, X-F_{n}\right\}$. Then given $x \in X$ there is $n \in N$ with $x \in F_{n} \subset s t\left(x, \mathscr{C}_{n}\right)=U_{n}$.

" $\Longleftarrow$ Let $\mathscr{Q}=\left\{U_{n}: n \in N\right\}$ be a countable open cover of countably $\sigma$-paracompact space $X$. Let $\left\{\mathscr{U}_{m}\right\}$ be a sequence of open covers of $X$ such that given $x \in X$ there are $U_{n} \in \mathscr{U}_{\text {and }} m \in N$ with $s t\left(x, \mathscr{U}_{m}\right) \subset$ $U_{n}$. We construct a countable closed refinement of $\mathscr{U}$ as follows.

Let $F_{m n}=\left\{x \in X: s t\left(x, \mathscr{U}_{m}\right) \subset U_{n}\right\}$. Then $\left\{F_{m n}: m, n \in N\right\}$ is a countable closed refinement of $\mathscr{W}$.

(i) Each $F_{m n}$ is contained in some element of $\mathscr{U}$ : clearly $F_{m n} \subset U_{n}$.

(ii) $\left\{F_{m n}: m, n \in N\right\}$ covers $X$ : Given $x \in X$, there are $m$ and $n$ such that $\operatorname{st}\left(x, \mathscr{U}_{m}\right) \subset U_{n}$, so $x \in F_{m n}$.

(iii) Each $F_{m n}$ is closed: To show $X-F_{m n}$ is open, let $y \in X-$ $F_{m n}$. Then $s t\left(y, \mathscr{U}_{m}\right) \not \subset U_{n}$, so there is a $U \in \mathscr{U}_{m}$ with $y \in U$, but $U \not \subset$ $U_{n}$. Then $U$ is an open neighborhood of $y$ not intersecting $F_{m n}$, for; if $z \in F_{m n}$ and $z \in U$, then $U \subset U_{n}$, which is not the case.

It is known, as previously mentioned, that countable subparacompactness and countable metacompactness are equivalent in normal spaces. The question arises, can we weaken normality and still get 
equivalence? We find an affirmative answer, and, in fact, arrive at another characterization of countably subparacompact spaces, by defining subnormality as follows.

Definition 2.4. $X$ is subnormal iff every finite open cover of $X$ has a countable closed refinement.

To see that every normal space is subnormal, recall that $X$ is normal iff every finite open cover of $X$ has a finite closed refinement. Fortuitously, every countably subparacompact space is also subnormal, as may be seen from Theorem 2.1(d). We may now state:

THEOREM 2.5. $X$ is countably subparacompact iff $X$ is countably metacompact and subnormal.

Proof of Theorem 2.5 requires the use of a characterization of countably metacompact spaces due to F. Ishikawa [4]. That is, $X$ is countably metacompact iff given a decreasing sequence $\left\{H_{n}\right\}$ of closed subsets of $X$ such that $\bigcap\left\{H_{n}\right\}=\varnothing$, there is a decreasing sequence $\left\{V_{n}\right\}$ of open sets in $X$ such that $H_{n} \subset V_{n}$ for all $n \in N$ and $\bigcap\left\{V_{n}\right\}=\varnothing$.

Proof of Theorem 2.5. " $\Longrightarrow$ " This follows directly from the cited result of Hodel and a remark following Definition 2.4.

"£" Let $\mathscr{C}=\left\{U_{n}: n \in N\right\}$ be a countable open cover of $X$. We shall construct a countable closed refinement $\mathscr{F}$ of $\mathscr{C}$. For each $n \in N$ set $H_{n}=X-\bigcup_{j=1}^{n} U_{j}$. Then $\left\{H_{n}\right\}$ is a decreasing sequence of closed subsets of $X$ such that $\bigcap\left\{H_{n}\right\}=\varnothing$. By Ishikawa's result there is thus a decreasing sequence $\left\{V_{n}\right\}$ of open sets in $X$ such that $H_{n} \subset V_{n}$ for all $n \in N$ and $\bigcap\left\{V_{n}\right\}=\varnothing$.

For each $n$, it is easily seen that $\left\{U_{1}, \cdots, U_{n}, V_{n}\right\}$ is a finite open cover of $X$ (if $x \notin \bigcup_{j=1}^{n} U_{j}$, then by construction $x \in H_{n}$, so $x \in V_{n}$ since $H_{n} \subset V_{n}$ ). As $X$ is subnormal, we may let $\mathscr{F}_{n}^{\prime}$ be a countable closed refinement of that cover.

Set $\mathscr{F}_{n}=\left\{F \cap\left(X-V_{n}\right): F \in \mathscr{F}_{n}^{\prime}\right\}$ and $\mathscr{F}=\bigcup_{n \in N} \mathscr{F}_{n}$. Then $\mathscr{F}$ is the required refinement, for:

(i) $\mathscr{F}$ is clearly a countable collection of closed sets in $X$.

(ii) Each element of $\mathscr{F}$ is contained in some element of $\mathscr{U}$ :

\footnotetext{
1 This proof is due to Phillip Zenor. The original was inelegant.
} 
If $F \in \mathscr{F}$, then $F \in \mathscr{F}_{n}$ for some $n \in N$. Thus $F$ is contained in $X-V_{n}$ and in some element of $\left\{U_{1}, \cdots, U_{n}, V_{n}\right\}$.

Clearly $F$ cannot be contained in $V_{n}$, so $F$ is contained in one of $\left\{U_{1}, \cdots, U_{n}\right\}$.

(iii) $\mathscr{F}$ covers $X$ : Let $x \in X$. Pick $n$ so that $x \notin V_{n}$. There is an element $F$ of $\mathscr{F}_{n}^{\prime}$ with $x \in F$. Clearly $x \in F \cap\left(X-V_{n}\right) \in \mathscr{F} \cdot \square$

3. Pathology. We have seen that countable subparacompactness is linked with normality via subnormality, which generalizes both. We shall see now that the pathological behavior of countable subparacompactness is similar to that of normality in products, and hence in inverse image theorems.

EXAMPLE 3.1. Let $W$ be the well known space consisting of all ordinals less than $\Omega$, the first uncountable ordinal. Let $W^{*}$ be $W \cup$ $\{\Omega\}$. Both $W$ and $W^{*}$ are given the order topology. $W^{*}$ is known to be a compact $T_{2}$ space and $W$ a countably compact, normal $T_{2}$ space. We shall show $W \times W^{*}$ is not subnormal (it has been known for some time that $W \times W^{*}$ is not normal). For a good presentation of $W$ and $W^{*}$, look up "ordinal examples" in the index of Greever's book [2].

Three facts about $W$ and $W^{*}$ given in the next lemma will be needed. An outline of the proof of this lemma is given in [5, Problem $4 E$ ].

Lemma 3.2. (a) If $A$ is a countable subset of $W$, then sup $(A)$ exists and belongs to $W$.

(b) If $x \in W^{*}$ and $x>1$, then $\{(\alpha, x]: \alpha<x\}$ is a fundamental system of open neighborhoods of $x . \quad\{1\}$ is itself an open set in $W^{*}$.

(c) If $\left\{x_{n}\right\}$ and $\left\{y_{n}\right\}$ are sequences in $W$ such that $x_{n} \leqq y_{n} \leqq x_{n+1}$ for all $n \in N$, then there is an element $z$ of $W$ such that $\left\{x_{n}\right\}$ and $\left\{y_{n}\right\}$ both converge to $z$.

Verification that $W \times W^{*}$ is not subnormal:

Let $H=\{(x, \Omega): x \in W\}$ and $K=\{(x, x): x \in W\} . \quad H$ and $K$ are disjoint closed sets in $W \times W^{*}$, so $\left\{\left(W \times W^{*}\right)-H,\left(W \times W^{*}\right)-K\right\}$ is an open cover of $W \times W^{*}$. Suppose, as is false, that there is a countable closed refinement $\mathscr{C}$ of this cover. Call the elements of $\mathscr{C} F_{n}$ if they intersect $H$ and $G_{n}$ if they do not intersect $H$.

For each $x>1$ in $W$ and each $n \in N,(x, \Omega) \notin G_{n}$, so there are $z_{n x}$ and $y_{n x}$ such that both are in $W$ and $\left(z_{n x}, x\right] \times\left(y_{n x}, \Omega\right] \cap G_{n}=\varnothing$. If $x=1$, there is $y_{n x}$ such that $\{1\} \times\left(y_{n x}, \Omega\right] \cap G_{n}=\varnothing$. 
Let $y_{x}=\sup \left\{y_{n x}: n \in N\right\}$. Then $\left\{(x, y): y \in W^{*}, y>y_{x}\right\}$ is disjoint from $G_{n}$ for all $n \in N$ and nonempty because $y_{x} \in W$ by 3.2(a).

Assertion. For some $n_{0} \in N$, given $x \in W$ there are $w$ and $y$ in $W$ with $x<w<y$ and $(w, y) \in F_{n_{0}}$.

Proof of the Assertion. Suppose it were false, then for each $n$, there would be an $x_{n}$ in $W$ such that $(w, y) \notin F_{n}$ whenever $x_{n}<w<y$. We could then let $x_{0}=\sup \left\{x_{n}: n \in N\right)$ and pick $y_{0}>y_{x_{0}}$ in $W$. The point $\left(x_{0}, y_{0}\right)$ would belong to no $F_{n}$ or $G_{n}$, an impossibility.

Construct a sequence $\left\{\left(x_{n}, y_{n}\right)\right\}$ in $(W \times W) \cap F_{n_{0}}$ as follows. Let $\left(x_{1}, y_{1}\right)$ be any point in $(W \times W) \cap F_{n_{0}}$ with $y_{1}>x_{1}$. In general, pick $\left(x_{n+1}, y_{n+1}\right)$ in $(W \times W) \cap F_{n_{0}}$ with $y_{n}<x_{n+1}<y_{n+1}$. The assertion assures us we can do this.

Then $\left\{x_{n}\right\}$ and $\left\{y_{n}\right\}$ are sequences in $W$ such that $x_{n} \leqq y_{n} \leqq x_{n+1}$ for all $n \in N$. By 3.2(c) there is $z \in W$ such that $\left\{x_{n}\right\}$ and $\left\{y_{n}\right\}$ converge to $z$. Thus $(z, z)$ is a limit point of $F_{n_{0}}$, implying $(z, z) \in F_{n_{0}}$. Hence $K \cap F_{n_{0}} \neq \varnothing$, a contradiction.

Example 3.1 shows that Theorem 3.3 of Singal and Jain [8] is false; i.e., it is not true that if $f: X \rightarrow Y$ is a closed, continuous mapping from a regular space $X$ onto a countably subparacompact space $Y$ such that $f^{-1}(y)$ is compact for each $y \in Y$, then $X$ is countably subparacompact. The mistake in their proof lies in the nextto-last sentence, which is untrue.

A number of product and inverse image theorems for countably subparacompact spaces are given in [6].

\section{REFERENCES}

1. D. K. Burke, On subparacompact spaces, Proc. Amer. Math. Soc., 23 (1969), 655-663.

2. J. Greever, Theory and Examples of Point Set Topology, California: Brooks/Cole Publishing Co., 1967.

3. R. E. Hodel, A note on subparacompact spaces, Proc. Amer. Math. Soc., 25 (1970), 842-845.

4. F. Ishikawa, On countably paracompact spaces, Proc. Japan Academy, 31 (1955), 686687.

5. J. Kelley, General Topology, New York: D. Van Nostrand Co., 1955.

6. T. R. Kramer, Countably Subparacompact Spaces, unpublished, 1972.

7. M. J. Mansfield, On countably paracompact normal spaces, Canad. J. Math., 9 (1957), 443-449.

8. M. K. Singal and P. Jain, On Subparacompact and Countably Subparacompact Spaces, Bull. Austral. Math. Soc., 5 (1971), 289-304.

Received January 25, 1972 and in revised form June 15, 1972. This paper represents a portion of a doctoral dissertation written under the direction of Dr. R. E. Hodel at Duke University. 



\section{PACIFIC JOURNAL OF MATHEMATICS}

\section{EDITORS}

D. Gilbarg and J. Milgram Stanford University

Stanford, California 94305

R. A. Beaumont

University of Washington

Seattle, Washington 98105
J. DugundJI

Department of Mathematics

University of Southern California

Los Angeles, California 90007

RICHARD ARENS

University of California

Los Angeles, California 90024

ASSOCIATE EDITORS
E. F. BECKENBACH
B. H. NeumanN
F. WOLF
K. YoSHIDA

\section{SUPPORTING INSTITUTIONS}

UNIVERSITY OF BRITISH COLUMBIA

CALIFORNIA INSTITUTE OF TECHNOLOGY

UNIVERSITY OF CALIFORNIA

MONTANA STATE UNIVERSITY

UNIVERSITY OF NEVADA

NEW MEXICO STATE UNIVERSITY

OREGON STATE UNIVERSITY

UNIVERSITY OF OREGON

OSAKA UNIVERSITY
UNIVERSITY OF SOUTHERN CALIFORNIA

STANFORD UNIVERSITY

UNIVERSITY OF TOKYO

UNIVERSITY OF UTAH

WASHINGTON STATE UNIVERSITY

UNIVERSITY OF WASHINGTON

$\stackrel{*}{*} \stackrel{*}{*} \stackrel{*}{*}$ AMERICAN MATHEMATICAL SOCIETY

NAVAL WEAPONS CENTER 


\section{Pacific Journal of Mathematics}

\section{Vol. 46, No. $1 \quad$ November, 1973}

Allan Francis Abrahamse, Uniform integrability of derivatives on $\sigma$-lattices .......................................... 1

Ronald Alter and K. K. Kubota, The diophantine equation $x^{2}+D=p^{n} \ldots \ldots \quad 11$

Grahame Bennett, Some inclusion theorems for sequence spaces .......... 17

William Cutler, On extending isotopies ........................ 31

Robert Jay Daverman, Factored codimension one cells in Euclidean

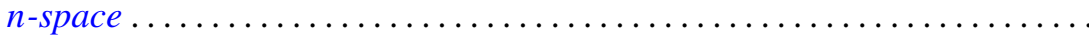

Patrick Barry Eberlein and Barrett O’Neill, Visibility manifolds ............ 45

M. Edelstein, Concerning dentability .......................... 111

Edward Graham Evans, Jr., Krull-Schmidt and cancellation over local

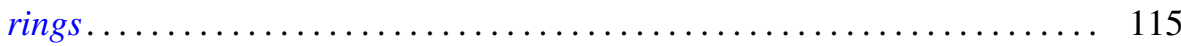

C. D. Feustel, A generalization of Kneser's conjecture ................ 123

Avner Friedman, Uniqueness for the Cauchy problem for degenerate parabolic equations .......................................... 131

David Golber, The cohomological description of a torus action ............ 149

Alain Goullet de Rugy, Un théorème du genre "Andô-Edwards" pour les

Fréchet ordonnés normaux..............................

Louise Hay, The class of recursively enumerable subsets of a recursively enumerable set ........................................

John Paul Helm, Albert Ronald da Silva Meyer and Paul Ruel Young, On orders of translations and enumerations...

Julien O. Hennefeld, A decomposition for $B(X)^{*}$ and unique Hahn-Banach

extensions

Gordon G. Johnson, Moment sequences in Hilbert space .

Thomas Rollin Kramer, A note on countably subparacompact spaces ...

Yves A. Lequain, Differential simplicity and extensions of a derivation ....

Peter Lorimer, A property of the groups Aut $\mathrm{PU}\left(3, q^{2}\right) \ldots$

225

Yasou Matsugu, The Levi problem for a product manifold.

231

John M.F. O'Connell, Real parts of uniform algebras ......

William Lindall Paschke, A factorable Banach algebra without bounded

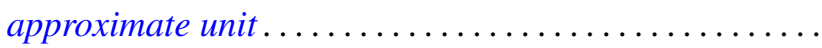

Ronald Joel Rudman, On the fundamental unit of a purely cubic field ....

Tsuan Wu Ting, Torsional rigidities in the elastic-plastic torsion of simply connected cylindrical bars .........................

Philip C. Tonne, Matrix representations for linear transformations on analytic sequences...................................

Jung-Hsien Tsai, On E-compact spaces and generalizations of perfect

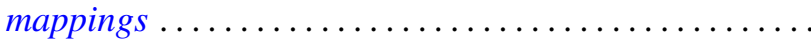

Alfons Van Daele, The upper envelope of invariant functionals majorized by an invariant weight. .. 